\title{
Analysis of fractional boundary value problem with non local flux multi-point conditions on a Caputo fractional differential equation
}

\author{
Muthaiah Subramanian, A Ramamurthy Vidhya Kumar and Thangaraj \\ Nandha Gopal
}

\begin{abstract}
A brief analysis of boundary value problem of Caputo fractional differential equation with nonlocal flux multi-point boundary conditions has been done. The investigation depends on the Banach fixed point theorem, KrasnoselskiiSchaefer fixed point theorem due to Burton and Kirk, fixed point theorem due to O'Regan. Relevant examples illustrating the main results are also constructed.
\end{abstract}

Mathematics Subject Classification (2010): 34A08, 34A12, 34B10.

Keywords: Fractional differential equation, Caputo derivative, multi-point, nonlocal, integral conditions, existence, fixed point.

\section{Introduction}

In recent years, fractional differential equations are increasingly utilized to model many problems in biology, chemistry, engineering, physics, economic and other areas of applications. The fractional differential equations have become a useful tool for describing nonlinear phenomena of science and engineering models. Also, researchers found that fractional calculus was very suitable to describe long memory and hereditary properties of various materials and processes. we refer the reader to the texts [16]-[14], [8], [9]-[6], and the references cited therein.

Fractional differential equations have attracted considerable interest because of their ability to model complex artefacts. These equations capture non local relations in space and time with memory essentials. Due to extensive applications of FDEs in engineering and science, research in this area has grown significantly all around the world., for instance, see [18], [11], [15] and the references cited therein. Recently, much interest has been created in establishing the existence of solutions for various types of 
boundary value problem of fractional order with nonlocal multi-point boundary conditions. Nonlocal multi-point conditions involving Liouville-Caputo derivative, first of its kind was explored by Agarwal et.al. [1] on nonlinear fractional order boundary value problem. Ahmad et.al. [2]-[5], [3], [7] profound the idea of new kind of nonlocal multi-point boundary value problem of fractional integro-differential equations involving multi-point strips integral boundary conditions.

In this paper the existence and uniqueness of solutions for the below fractional differential equations with nonlocal multi-point boundary conditions are discussed. Consider the fractional differential equation

$$
C_{\mathfrak{D}}{ }^{\delta} p(z)=k(z, p(z)), \quad z \in \mathfrak{J}=[0,1], \quad n-1<\delta \leq n,
$$

supplemented with the nonlocal multi-point integral boundary conditions

$$
\begin{aligned}
& p(0)=\psi(p), p^{\prime}(0)=\rho p^{\prime}(\nu), p^{\prime \prime}(0)=0, p^{\prime \prime \prime}(0)=0, \cdots, p^{n-2}(0)=0, \\
& p(1)=\lambda \int_{0}^{\varsigma} p(\sigma) d \sigma+\mu \sum_{j=1}^{m-2} \xi_{j} p\left(\zeta_{j}\right),
\end{aligned}
$$

where ${ }^{C} \mathfrak{D}^{\delta}$ denote the Caputo fractional derivative and $k: \mathfrak{J} \times \mathbb{R}$ to $\mathbb{R}$ and $\psi: C(\mathfrak{J}, \mathbb{R})$ to $\mathbb{R}$, are given continuous functions, $0<\nu<\varsigma<\zeta_{1}<\zeta_{2}<\cdots<\zeta_{m-2}<1$, $\xi_{j}, j=1,2, \cdots, m-2, \rho, \lambda, \mu$ are positive real constants. The rest of the paper is organised as follows: The preliminaries section is devoted to some fundamental concepts of fractional calculus with basic lemma related to the given problem. In section 3, the existence and uniqueness of solutions are obtained based on Banach fixed point theorem, Krasnoselskii-Schaefer fixed point theorem due to Burton and Kirk, and fixed point theorem due to O'Regan and also the validation of the results is done by providing examples.

\section{Preliminaries}

In this section, we introduce some notations and definitions of fractional calculus.

Definition 2.1. The fractional integral of order $\delta$ with the lower limit zero for a function $k$ is defined as

$$
\Im^{\delta} k(z)=\frac{1}{\Gamma(\delta)} \int_{0}^{z} \frac{k(\sigma)}{(z-\sigma)^{1-\delta}} d \sigma, \quad z>0, \delta>0,
$$

provided the right hand-side is point-wise defined on $[0, \infty)$, where $\Gamma(\cdot)$ is the gamma function, which is defined by $\Gamma(\delta)=\int_{0}^{\infty} z^{\delta-1} e^{-z} d z$.

Definition 2.2. The Riemann-Liouville fractional derivative of order $\delta>0, n-1<$ $\delta<n, n \in \mathbb{N}$ is defined as

$$
\mathfrak{D}_{0+}^{\delta} k(z)=\frac{1}{\Gamma(n-\delta)}\left(\frac{d}{d z}\right)^{n} \int_{0}^{z}(z-\sigma)^{n-\delta-1} k(\sigma) d \sigma,
$$

where the function $k(z)$ has absolutely continuous derivative up to order $(n-1)$. 
Definition 2.3. The Caputo derivative of order $\delta$ for a function $k:[0, \infty) \rightarrow \mathbb{R}$ can be written as

$$
C_{\mathfrak{D}^{\delta}} k(z)=\mathfrak{D}_{0+}^{\delta}\left(k(z)-\sum_{j=0}^{n-1} \frac{z^{j}}{j !} k^{(j)}(0)\right), \quad z>0, \quad n-1<\delta<n .
$$

Remark 2.4. If $k(z) \in C^{n}[0, \infty)$, then

$$
\begin{aligned}
{ }^{C} \mathfrak{D}^{\delta} k(z) & =\frac{1}{\Gamma(n-\delta)} \int_{0}^{z} \frac{k^{n}(\sigma)}{(z-\sigma)^{\delta+1-n}} d \sigma \\
& =\mathfrak{I}^{n-\delta} k^{n}(z), \quad z>0, n-1<\delta<n .
\end{aligned}
$$

Lemma 2.5. For $\delta>0$, the general solution of the fractional differential equation $C \mathfrak{D}^{\delta} p(z)=0$ is given by

$$
p(z)=a_{0}+a_{1} z+\cdots+a_{n-1} z^{n-1},
$$

where $a_{i} \in \mathbb{R}, i=1,2, \ldots, n-1(n=[\delta]+1)$.

In view of Lemma 2.5, it follows that

$$
\mathfrak{I}^{\delta C} \mathfrak{D}^{\delta} p(z)=p(z)+a_{0}+a_{1} z+\cdots+a_{n-1} z^{n-1},
$$

for some $a_{i} \in \mathbb{R}, i=1,2, \ldots, n-1(n=[\delta]+1)$.

Next, we present an auxiliary lemma which plays a key role in the sequel.

Lemma 2.6. For any $\hat{k} \in C(\mathfrak{J}, \mathbb{R})$, the solution of the linear fractional differential equation

$$
{ }^{C} \mathfrak{D}^{\delta} p(z)=\hat{k}(z), \quad n-1<\delta \leq n,
$$

supplemented with the boundary conditions (1.2) is given by

$$
\begin{aligned}
p(z)= & \int_{0}^{z} \frac{(z-\sigma)^{\delta-1}}{\Gamma(\delta)} \hat{k}(\sigma) d \sigma \\
& +\left[1+\frac{\left(z v_{1}+z^{n-1} \varpi_{1}\right)}{\vartheta}\left(\lambda \delta+\mu \sum_{j=1}^{m-2} \xi_{j}-1\right)\right] \psi(p) \\
& +\frac{\rho\left(z v_{2}-z^{n-1} \varpi_{2}\right)}{\vartheta}\left[\int_{0}^{\nu} \frac{(\nu-\sigma)^{\delta-2}}{\Gamma(\delta-1)} \hat{k}(\sigma) d \sigma\right] \\
& +\frac{\left(z v_{1}+z^{n-1} \varpi_{1}\right)}{\vartheta}\left[\lambda \int_{0}^{\varsigma}\left(\int_{0}^{\sigma} \frac{(\sigma-\theta)^{\delta-1}}{\Gamma(\delta)} \hat{k}(\theta) d \theta\right) d \sigma\right. \\
& \left.+\mu \sum_{j=1}^{m-2} \xi_{j} \int_{0}^{\zeta_{j}} \frac{\left(\zeta_{j}-\sigma\right)^{\delta-1}}{\Gamma(\delta)} \hat{k}(\sigma) d \sigma-\int_{0}^{1} \frac{(1-\sigma)^{\delta-1}}{\Gamma(\delta)} \hat{k}(\sigma) d \sigma\right]
\end{aligned}
$$


where

$$
\begin{aligned}
\varpi_{1} & =1-\rho, \quad \varpi_{2}=1-\frac{\lambda \delta^{2}}{2}-\mu \sum_{j=1}^{m-2} \xi_{j} \zeta_{j} \\
v_{1} & =(n-1) \rho \delta^{n-2}, \quad v_{2}=1-\frac{\lambda \delta^{n}}{n}-\mu \sum_{j=1}^{m-2} \xi_{j} \zeta_{j}^{n-1} \\
\vartheta & =\varpi_{1} v_{2}+\varpi_{2} v_{1} \neq 0,
\end{aligned}
$$

Proof. It is evident that the general solution of the fractional differential equations in (2.1) can be written as

$$
p(z)=\int_{0}^{z} \frac{(z-\sigma)^{\delta-1}}{\Gamma(\delta)} \hat{k}(\sigma) d \sigma+a_{0}+a_{1} z+a_{2} z^{2}+\cdots+a_{n-1} z^{n-1}
$$

where $a_{i} \in \mathbb{R},(i=0,1,2, \ldots,(n-1))$ are arbitrary constants. Using the boundary conditions given by (1.2) in (2.6), we get $a_{0}=\psi(p)$. On using the notations (2.3)(2.5) along with (1.2) in (2.6), we get

$$
\begin{aligned}
a_{1} \varpi_{1}-a_{n-1} v_{1}= & \rho \int_{0}^{\nu} \frac{(\nu-\sigma)^{\delta-2}}{\Gamma(\delta-1)} \hat{k}(\sigma) d \sigma \\
a_{1} \varpi_{2}+a_{n-1} v_{2}= & \lambda \int_{0}^{\varsigma}\left(\int_{0}^{\sigma} \frac{(\sigma-\theta)^{\delta-1}}{\Gamma(\delta)} \hat{k}(\theta) d \theta\right) d \sigma \\
& +\mu \sum_{j=1}^{m-2} \xi_{j} \int_{0}^{\zeta_{j}} \frac{\left(\zeta_{j}-\sigma\right)^{\delta-1}}{\Gamma(\delta)} \hat{k}(\sigma) d \sigma \\
& -\int_{0}^{1} \frac{(1-\sigma)^{\delta-1}}{\Gamma(\delta)} \hat{k}(\sigma) d \sigma .
\end{aligned}
$$

Solving the system (2.7) and (2.8) for $a_{1}, a_{n-1}$, we get

$$
\begin{aligned}
a_{1} & =\frac{1}{\vartheta}\left[v_{2}\left(\rho \int_{0}^{\nu} \frac{(\nu-\sigma)^{\delta-2}}{\Gamma(\delta-1)} \hat{k}(\sigma) d \sigma\right)\right. \\
& +v_{1}\left(\lambda \int_{0}^{\varsigma}\left(\int_{0}^{\sigma} \frac{(\sigma-\theta)^{\delta-1}}{\Gamma(\delta)} \hat{k}(\theta) d \theta\right) d \sigma\right. \\
& +\mu \sum_{j=1}^{m-2} \xi_{j} \int_{0}^{\zeta_{j}} \frac{\left(\zeta_{j}-\sigma\right)^{\delta-1}}{\Gamma(\delta)} \hat{k}(\sigma) d \sigma+\psi(p)\left(\lambda \delta+\mu \sum_{j=1}^{m-2} \xi_{j}-1\right) \\
& \left.\left.-\int_{0}^{1} \frac{(1-\sigma)^{\delta-1}}{\Gamma(\delta)} \hat{k}(\sigma) d \sigma\right)\right]
\end{aligned}
$$




$$
\begin{aligned}
a_{n-1} & =\frac{-1}{\vartheta}\left[\varpi_{2}\left(\rho \int_{0}^{\nu} \frac{(\nu-\sigma)^{\delta-2}}{\Gamma(\delta-1)} \hat{k}(\sigma) d \sigma\right)\right. \\
& +\varpi_{1}\left(\lambda \int_{0}^{\varsigma}\left(\int_{0}^{\sigma} \frac{(\sigma-\theta)^{\delta-1}}{\Gamma(\delta)} \hat{k}(\theta) d \theta\right) d \sigma\right. \\
& +\mu \sum_{j=1}^{m-2} \xi_{j} \int_{0}^{\zeta_{j}} \frac{\left(\zeta_{j}-\sigma\right)^{\delta-1}}{\Gamma(\delta)} \hat{k}(\sigma) d \sigma+\psi(p)\left(\lambda \delta+\mu \sum_{j=1}^{m-2} \xi_{j}-1\right) \\
& \left.\left.-\int_{0}^{1} \frac{(1-\sigma)^{\delta-1}}{\Gamma(\delta)} \hat{k}(\sigma) d \sigma\right)\right] .
\end{aligned}
$$

Substituting the values of $a_{0}, a_{1}, a_{n-1}$ in (2.6), we get the solution (2.2). This completes the proof.

\section{Main results}

We denote by $\mathfrak{G}=C(\mathfrak{J}, \mathbb{R})$ be the Banach space of all continuous functions from $\mathfrak{J} \rightarrow \mathbb{R}$, equipped with the norm defined by

$$
\left.\|p\|=\sup _{z \in \mathfrak{J}}|p(z)|, z \in \mathfrak{J}\right\} .
$$

Also by $\mathfrak{L}^{1}(\mathfrak{J}, \mathbb{R})$, we denote the Banach space of measurable functions $p: \mathfrak{J} \rightarrow \mathbb{R}$ which are Lebesgue integral and normed by

$$
\|p\|_{\mathfrak{L}^{1}}=\int_{0}^{1}|p(z)| d z .
$$

In view of Lemma 2.6, we define an operator $\mathfrak{T}: \mathfrak{G} \rightarrow \mathfrak{G}$ associated with problem (1.1) as

$$
\begin{aligned}
(\mathfrak{T} p)(z)= & \int_{0}^{z} \frac{(z-\sigma)^{\delta-1}}{\Gamma(\delta)} k(\sigma, p(\sigma)) d \sigma \\
& +\left[1+\frac{\left(z v_{1}+z^{n-1} \varpi_{1}\right)}{\vartheta}\left(\lambda \delta+\mu \sum_{j=1}^{m-2} \xi_{j}-1\right)\right] \psi(p) \\
& +\frac{\rho\left(z v_{2}-z^{n-1} \varpi_{2}\right)}{\vartheta}\left[\int_{0}^{\nu} \frac{(\nu-\sigma)^{\delta-2}}{\Gamma(\delta-1)} k(\sigma, p(\sigma)) d \sigma\right] \\
& +\frac{\left(z v_{1}+z^{n-1} \varpi_{1}\right)}{\vartheta}\left[\lambda \int_{0}^{\varsigma}\left(\int_{0}^{\sigma} \frac{(\sigma-\theta)^{\delta-1}}{\Gamma(\delta)} k(\theta, p(\theta)) d \theta\right) d \sigma\right. \\
& +\mu \sum_{j=1}^{m-2} \xi_{j} \int_{0}^{\zeta_{j}} \frac{\left(\zeta_{j}-\sigma\right)^{\delta-1}}{\Gamma(\delta)} k(\sigma, p(\sigma)) d \sigma \\
& \left.-\int_{0}^{1} \frac{(1-\sigma)^{\delta-1}}{\Gamma(\delta)} k(\sigma, p(\sigma)) d \sigma\right]
\end{aligned}
$$


Let us define $\mathfrak{T}_{1}, \mathfrak{T}_{2}: \mathfrak{G} \rightarrow \mathfrak{G}$ by

$$
\begin{aligned}
\left(\mathfrak{T}_{1} p\right)(z)= & \int_{0}^{z} \frac{(z-\sigma)^{\delta-1}}{\Gamma(\delta)} k(\sigma, p(\sigma)) d \sigma \\
& +\frac{\rho\left(z v_{2}-z^{n-1} \varpi_{2}\right)}{\vartheta}\left[\int_{0}^{\nu} \frac{(\nu-\sigma)^{\delta-2}}{\Gamma(\delta-1)} k(\sigma, p(\sigma)) d \sigma\right] \\
& +\frac{\left(z v_{1}+z^{n-1} \varpi_{1}\right)}{\vartheta}\left[\lambda \int_{0}^{\varsigma}\left(\int_{0}^{\sigma} \frac{(\sigma-\theta)^{\delta-1}}{\Gamma(\delta)} k(\theta, p(\theta)) d \theta\right) d \sigma\right. \\
& +\mu \sum_{j=1}^{m-2} \xi_{j} \int_{0}^{\zeta_{j}} \frac{\left(\zeta_{j}-\sigma\right)^{\delta-1}}{\Gamma(\delta)} k(\sigma, p(\sigma)) d \sigma \\
& \left.-\int_{0}^{1} \frac{(1-\sigma)^{\delta-1}}{\Gamma(\delta)} k(\sigma, p(\sigma)) d \sigma\right]
\end{aligned}
$$

and

$$
\left(\mathfrak{T}_{2} p\right)(z)=\left[1+\frac{\left(z v_{1}+z^{n-1} \varpi_{1}\right)}{\vartheta}\left(\lambda \delta+\mu \sum_{j=1}^{m-2} \xi_{j}-1\right)\right] \psi(p)
$$

In the sequel, we use the notations:

$$
\widehat{\eta}=\frac{1}{\Gamma(\delta+1)}\left[1+\frac{\rho\left|\left(v_{2}-\varpi_{2}\right)\right| \nu^{\delta-1}}{\vartheta \delta}+\frac{\left(v_{1}+\varpi_{1}\right)}{\vartheta}\left(\frac{\lambda \varsigma^{\delta+1}}{\delta+1}+\mu \sum_{j=1}^{m-2} \xi_{j} \zeta_{j}^{\delta}+1\right)\right]
$$

and

$$
\widehat{\omega}=1+\frac{\left(v_{1}+\varpi_{1}\right)}{\vartheta}\left(\lambda \delta+\mu \sum_{j=1}^{m-2} \xi_{j}+1\right)
$$

Theorem 3.1. The continuous function $k$ defined from $\mathfrak{J} \times \mathbb{R}$ to $\mathbb{R}$. Let us speculate that

$\left(\mathfrak{E}_{1}\right)|k(z, p)-k(z, q)| \leq \mathfrak{S}\|p-q\|, \forall z \in \mathfrak{J}, \mathfrak{S}>0, p, q \in \mathbb{R}$.

$\left(\mathfrak{E}_{2}\right)$ The continuous function $\psi$ defined from $C(\mathfrak{J}, \mathbb{R}) \rightarrow \mathbb{R}$ satisfying the condition: $|\psi(v)-\psi(w)| \leq \varepsilon\|v-w\|, \varepsilon \widehat{\omega}<1, \forall v, w \in C(\mathfrak{J}, \mathbb{R}), \varepsilon>0$.

$\left(\mathfrak{E}_{3}\right) \quad \Theta:=\mathfrak{S} \widehat{\eta}+\varepsilon \widehat{\omega}<1$. Then the boundary value problem (1.1)-(1.2) has unique solution on $\mathfrak{J}$.

Proof. For $p, q \in \mathfrak{G}$ and for each $z \in \mathfrak{J}$, from the definition of $\mathfrak{T}$ and assumptions $\left(\mathfrak{E}_{1}\right)$ and $\left(\mathfrak{E}_{2}\right)$. We obtain

$$
\begin{gathered}
|(\mathfrak{T} p)(z)-(\mathfrak{T} q)(z)| \leq \sup _{z \in \mathfrak{J}}\left\{\int_{0}^{z} \frac{(z-\sigma)^{\delta-1}}{\Gamma(\delta)}|k(\sigma, p(\sigma))-k(\sigma, q(\sigma))| d \sigma\right. \\
+\left|\left[1+\frac{\left(z v_{1}+z^{n-1} \varpi_{1}\right)}{\vartheta}\left(\lambda \delta+\mu \sum_{j=1}^{m-2} \xi_{j}-1\right)\right]\right||\psi(p)-\psi(q)| \\
+\left|\frac{\rho\left(z v_{2}-z^{n-1} \varpi_{2}\right)}{\vartheta}\right|\left[\int_{0}^{\nu} \frac{(\nu-\sigma)^{\delta-2}}{\Gamma(\delta-1)}|k(\sigma, p(\sigma))-k(\sigma, q(\sigma))| d \sigma\right]
\end{gathered}
$$




$$
\begin{aligned}
& +\left|\frac{\left(z v_{1}+z^{n-1} \varpi_{1}\right)}{\vartheta}\right|\left[\lambda \int_{0}^{\varsigma}\left(\int_{0}^{\sigma} \frac{(\sigma-\theta)^{\delta-1}}{\Gamma(\delta)}|k(\theta, p(\theta))-k(\theta, q(\theta))| d \theta\right) d \sigma\right. \\
& +\mu \sum_{j=1}^{m-2} \xi_{j} \int_{0}^{\zeta_{j}} \frac{\left(\zeta_{j}-\sigma\right)^{\delta-1}}{\Gamma(\delta)}|k(\sigma, p(\sigma))-k(\sigma, q(\sigma))| d \sigma \\
& \left.\left.+\int_{0}^{1} \frac{(1-\sigma)^{\delta-1}}{\Gamma(\delta)}|k(\sigma, p(\sigma))-k(\sigma, q(\sigma))| d \sigma\right]\right\} \\
& \leq \int_{0}^{z} \frac{(z-\sigma)^{\delta-1}}{\Gamma(\delta)}(\mathfrak{S}\|p-q\|) d \sigma \\
& +\left|\left[1+\frac{\left(z v_{1}+z^{n-1} \varpi_{1}\right)}{\vartheta}\left(\lambda \delta+\mu \sum_{j=1}^{m-2} \xi_{j}-1\right)\right]\right||\psi(p)-\psi(q)| \\
& +\left|\frac{\rho\left(z v_{2}-z^{n-1} \varpi_{2}\right)}{\vartheta}\right|\left[\int_{0}^{\nu} \frac{(\nu-\sigma)^{\delta-2}}{\Gamma(\delta-1)}(\mathfrak{S}\|p-q\|) d \sigma\right] \\
& +\left|\frac{\left(z v_{1}+z^{n-1} \varpi_{1}\right)}{\vartheta}\right|\left[\lambda \int_{0}^{\varsigma}\left(\int_{0}^{\sigma} \frac{(\sigma-\theta)^{\delta-1}}{\Gamma(\delta)}(\mathfrak{S}\|p-q\|) d \theta\right) d \sigma\right. \\
& \left.+\mu \sum_{j=1}^{m-2} \xi_{j} \int_{0}^{\zeta_{j}} \frac{\left(\zeta_{j}-\sigma\right)^{\delta-1}}{\Gamma(\delta)}(\mathfrak{S}\|p-q\|) d \sigma+\int_{0}^{1} \frac{(1-\sigma)^{\delta-1}}{\Gamma(\delta)}(\mathfrak{S}\|p-q\|) d \sigma\right] \\
& \leq \frac{\mathfrak{S}}{\Gamma(\delta+1)}\left[1+\frac{\rho\left|\left(v_{2}-\varpi_{2}\right)\right| \nu^{\delta-1}}{\vartheta \delta}+\frac{\left(v_{1}+\varpi_{1}\right)}{\vartheta}\left(\frac{\lambda \varsigma^{\delta+1}}{\delta+1}+\mu \sum_{j=1}^{m-2} \xi_{j} \zeta_{j}^{\delta}+1\right)\right]\|p-q\| \\
& +\left[1+\frac{\left(v_{1}+\varpi_{1}\right)}{\vartheta}\left(\lambda \delta+\mu \sum_{j=1}^{m-2} \xi_{j}+1\right)\right] \varepsilon\|p-q\| \leq(\mathfrak{S} \widehat{\eta}+\varepsilon \widehat{\omega})\|p-q\| .
\end{aligned}
$$

Hence

$$
\|(\mathfrak{T} p)-(\mathfrak{T} q)\| \leq \Theta\|p-q\| .
$$

As $\Theta<1$ by $\left(\mathfrak{E}_{3}\right)$, the operator $\mathfrak{T}: \mathfrak{G} \rightarrow \mathfrak{G}$ is a contraction. Hence the conclusion of the theorem follows by the Banach fixed point theorem.

Example 3.2. Consider the fractional differential equation given by

$$
C_{\mathfrak{D}^{\frac{7}{3}}} p(z)=\sin z+\frac{e^{-z} \sin p(z)}{4 \sqrt{z^{6}+16}}, \quad z \in \mathfrak{J},
$$

subject to the boundary conditions

$$
p(0)=\frac{1}{10} p(z), p^{\prime}(0)=\frac{1}{4} x^{\prime}\left(\frac{1}{5}\right) p(1)=\int_{0}^{\frac{1}{3}} p(\sigma) d \sigma+\sum_{j=1}^{4} \xi_{j} p\left(\zeta_{j}\right) .
$$

Here

$$
\begin{gathered}
2<\delta \leq 3, \lambda=\mu=1, \rho=\frac{1}{4}, \nu=\frac{1}{5}, \varsigma=\frac{1}{3}, \\
\xi_{1}=\frac{1}{5}, \xi_{2}=\frac{1}{7}, \xi_{3}=\frac{1}{6}, \xi_{4}=\frac{1}{8}
\end{gathered}
$$




$$
\zeta_{1}=\frac{1}{2}, \zeta_{2}=\frac{1}{4}, \zeta_{3}=\frac{1}{3}, \zeta_{4}=\frac{1}{5} .
$$

Using the given data, we find that

$$
|k(z, p(z))|=\sin z+\frac{e^{-z} \sin p(z)}{4 \sqrt{z^{6}+16}}, \quad \psi(p)=\frac{1}{10} p(z) .
$$

Since

$$
\begin{gathered}
\mid k(z, p)-k(z, q) \leq \frac{1}{16}\|p-q\|, \\
|\psi(v)-\psi(w)| \leq \frac{1}{10}\|v-w\|,
\end{gathered}
$$

therefore, $\left(\mathfrak{E}_{1}\right)$ and $\left(\mathfrak{E}_{2}\right)$ are respectively satisfied with $\mathfrak{S}=\frac{1}{16}$ and $\varepsilon=\frac{1}{10}$. With the given data, we find that $\widehat{\eta}=5.18462, \widehat{\omega}=2.62014$, it is found that

$$
\Theta:=\mathfrak{S} \widehat{\eta}+\varepsilon \widehat{\omega} \cong 0.586053<1 .
$$

Thus, the assumptions of Theorem 3.1 hold and the problem (3.6)-(3.7) has at most one solution on $\mathfrak{J}$.

Theorem 3.3. Let $\mathfrak{Y}$ be a Banach space, and $\mathfrak{H}_{1}, \mathfrak{H}_{2}: \mathfrak{Y} \rightarrow \mathfrak{Y}$ be two operators such that $\mathfrak{H}_{1}$ is a contraction and $\mathfrak{H}_{2}$ is completely continuous. Then either

(i) the operator equation $u=\mathfrak{H}_{1}(u)+\mathfrak{H}_{2}(u)$ has a solution, or

(ii) the set $\mathfrak{F}=\left\{w \in \mathfrak{Y}: \kappa \mathfrak{H}_{1}\left(\frac{w}{\kappa}\right)+\kappa \mathfrak{H}_{2}(w)=w\right\}$ is unbounded for $\kappa \in(0,1)$.

Theorem 3.4. The continuous function $k$ defined from $\mathfrak{J} \times \mathbb{R}$ to $\mathbb{R}$ and condition $\left(\mathfrak{E}_{2}\right)$ hold. Also let us understand that:

$\left(\mathfrak{E}_{4}\right) \psi(0)=0$.

$\left(\mathfrak{E}_{5}\right)$ there exists a function $x \in \mathfrak{L}^{1}\left(\mathfrak{J}, \mathbb{R}_{+}\right)$such that $|k(z, v)| \leq x(z)$, for almost everywhere each $z \in \mathfrak{J}$, and each $v \in \mathbb{R}$.

Then the problem (1.1)-(1.2) has at least one solution on $\mathfrak{J}$.

Proof. To transform the problem (1.1)-(1.2) into a fixed point problem. we consider the map $\mathfrak{T}: \mathfrak{G} \rightarrow \mathfrak{G}$ given by $(\mathfrak{T} p)(z)=\left(\mathfrak{T}_{1} p\right)(z)+\left(\mathfrak{T}_{2} p\right)(z), z \in \mathfrak{J}$, where $\mathfrak{T}_{1}$ and $\mathfrak{T}_{2}$ are defined by (3.2) and (3.3) respectively.

We shall show that the operators $\mathfrak{T}_{1}$ and $\mathfrak{T}_{2}$ satisfy all the conditions of Theorem 3.3 .

Step 1. The operator $\mathfrak{T}_{1}$ defined by (3.2) is continuous.

Let $p_{n} \subset \mathfrak{B}_{\theta}=\{p \in \mathfrak{G}:\|p\| \leq \theta\}$ with $\left\|p_{n}-p\right\| \rightarrow 0$.

Then the limit $\left\|p_{n}(z)-p(z)\right\| \rightarrow 0$ is uniformly valid on $\mathfrak{J}$. From the uniform continuity of $k(z, p)$ on the compact set $\mathfrak{J} \times[-\theta, \theta]$, it follows that $\left\|k\left(z, p_{n}(z)\right)-k(z, p(z))\right\| \rightarrow 0$ uniformly on $\mathfrak{J}$. Hence $\left\|\mathfrak{T}_{1} p_{n}-\mathfrak{T}_{1} p\right\| \rightarrow 0$ as $n \rightarrow \infty$ which implies that the operator $\mathfrak{T}_{1}$ is continuous.

Step 2. The operator $\mathfrak{T}_{1}$ maps bounded sets into bounded sets in $\mathfrak{G}$.

It is indeed enough to show that for any $\theta>0$ there exists a positive constant $\mathfrak{S}$ such that for each

$$
p \in \mathfrak{B}_{\theta}=\{p \in \mathfrak{G}:\|p\| \leq \theta\}
$$

we have

$$
\left\|\mathfrak{T}_{1} p\right\| \leq \mathfrak{Q}
$$


Let $p \in \mathfrak{B}_{\theta}$. Then

$$
\begin{aligned}
& \left\|\mathfrak{T}_{1} p\right\| \leq \int_{0}^{z} \frac{(z-\sigma)^{\delta-1}}{\Gamma(\delta)}|k(\sigma, p(\sigma))| d \sigma \\
& +\frac{\rho\left(z v_{2}-z^{n-1} \varpi_{2}\right)}{\vartheta}\left[\int_{0}^{\nu} \frac{(\nu-\sigma)^{\delta-2}}{\Gamma(\delta-1)}|k(\sigma, p(\sigma))| d \sigma\right] \\
& +\frac{\left(z v_{1}+z^{n-1} \varpi_{1}\right)}{\vartheta}\left[\lambda \int_{0}^{\varsigma}\left(\int_{0}^{\sigma} \frac{(\sigma-\theta)^{\delta-1}}{\Gamma(\delta)}|k(\theta, p(\theta))| d \theta\right) d \sigma\right. \\
& +\mu \sum_{j=1}^{m-2} \xi_{j} \int_{0}^{\zeta_{j}} \frac{\left(\zeta_{j}-\sigma\right)^{\delta-1}}{\Gamma(\delta)}|k(\sigma, p(\sigma))| d \sigma \\
& \left.+\int_{0}^{1} \frac{(1-\sigma)^{\delta-1}}{\Gamma(\delta)}|k(\sigma, p(\sigma))| d \sigma\right] \\
& \leq \int_{0}^{z} \frac{(z-\sigma)^{\delta-1}}{\Gamma(\delta)} x(\sigma) d \sigma \\
& +\frac{\rho\left(z v_{2}-z^{n-1} \varpi_{2}\right)}{\vartheta}\left[\int_{0}^{\nu} \frac{(\nu-\sigma)^{\delta-2}}{\Gamma(\delta-1)} x(\sigma) d \sigma\right] \\
& +\frac{\left(z v_{1}+z^{n-1} \varpi_{1}\right)}{\vartheta}\left[\lambda \int_{0}^{\varsigma}\left(\int_{0}^{\sigma} \frac{(\sigma-\theta)^{\delta-1}}{\Gamma(\delta)} x(\theta) d \theta\right) d \sigma\right. \\
& \left.+\mu \sum_{j=1}^{m-2} \xi_{j} \int_{0}^{\zeta_{j}} \frac{\left(\zeta_{j}-\sigma\right)^{\delta-1}}{\Gamma(\delta)} x(\sigma) d \sigma+\int_{0}^{1} \frac{(1-\sigma)^{\delta-1}}{\Gamma(\delta)} x(\sigma) d \sigma\right] \\
& \leq \frac{\|x\|}{\Gamma(\delta+1)}\left[1+\frac{\rho\left|\left(v_{2}-\varpi_{2}\right)\right| \nu^{\delta-1}}{\vartheta \delta}\right. \\
& \left.+\frac{\left(v_{1}+\varpi_{1}\right)}{\vartheta}\left(\frac{\lambda \varsigma^{\delta+1}}{\delta+1}+\mu \sum_{j=1}^{m-2} \xi_{j} \zeta_{j}^{\delta}+1\right)\right]:=\mathfrak{Q}
\end{aligned}
$$

Step 3. The operator $\mathfrak{T}_{1}$ maps bounded sets into equicontinuous sets in $\mathfrak{G}$. Let $\varrho_{1}, \varrho_{2} \in \mathfrak{J}$ with $\varrho_{1}<\varrho_{2}$ and $p \in \mathfrak{B}_{\theta}$, we obtain

$$
\begin{array}{r}
\left|\left(\mathfrak{T}_{1} p\right)\left(\varrho_{2}\right)-\left(\mathfrak{T}_{1} p\right)\left(\varrho_{1}\right)\right| \leq\left|\int_{0}^{\varrho_{1}} \frac{\left[\left(\varrho_{2}-\sigma\right)^{\delta-1}-\left(\varrho_{1}-\sigma\right)^{\delta-1}\right]}{\Gamma(\delta)} \times k(\sigma, p(\sigma)) d \sigma\right| \\
+\left|\int_{\varrho_{1}}^{\varrho_{2}} \frac{\left(\varrho_{2}-\sigma\right)^{\delta-1}}{\Gamma(\delta)} k(\sigma, p(\sigma)) d \sigma\right| \\
+\left|\frac{\rho\left(\left(\varrho_{2}-\varrho_{1}\right) v_{2}-\left(\varrho_{2}^{n-1}-\varrho_{1}^{n-1}\right) \varpi_{2}\right)}{\vartheta}\right|\left[\int_{0}^{\nu} \frac{(\nu-\sigma)^{\delta-2}}{\Gamma(\delta-1)}|k(\sigma, p(\sigma))| d \sigma\right] \\
+\frac{\left(\left(\varrho_{2}-\varrho_{1}\right) v_{1}+\left(\varrho_{2}^{n-1}-\varrho_{1}^{n-1}\right) \varpi_{1}\right)}{\vartheta}\left[\lambda \int_{0}^{\varsigma}\left(\int_{0}^{\sigma} \frac{(\sigma-\theta)^{\delta-1}}{\Gamma(\delta)}|k(\theta, p(\theta))| d \theta\right) d \sigma\right.
\end{array}
$$




$$
\begin{aligned}
& \left.+\mu \sum_{j=1}^{m-2} \xi_{j} \int_{0}^{\zeta_{j}} \frac{\left(\zeta_{j}-\sigma\right)^{\delta-1}}{\Gamma(\delta)}|k(\sigma, p(\sigma))| d \sigma+\int_{0}^{1} \frac{(1-\sigma)^{\delta-1}}{\Gamma(\delta)}|k(\sigma, p(\sigma))| d \sigma\right] \\
& \leq\left|\int_{0}^{\varrho_{1}} \frac{\left[\left(\varrho_{2}-\sigma\right)^{\delta-1}-\left(\varrho_{1}-\sigma\right)^{\delta-1}\right]}{\Gamma(\delta)} \times x(\sigma) d \sigma\right|+\left|\int_{\varrho_{1}}^{\varrho_{2}} \frac{\left(\varrho_{2}-\sigma\right)^{\delta-1}}{\Gamma(\delta)} x(\sigma) d \sigma\right| \\
& +\left|\frac{\rho\left(\left(\varrho_{2}-\varrho_{1}\right) v_{2}-\left(\varrho_{2}^{n-1}-\varrho_{1}^{n-1}\right) \varpi_{2}\right)}{\vartheta}\right|\left[\int_{0}^{\nu} \frac{(\nu-\sigma)^{\delta-2}}{\Gamma(\delta-1)}|x(\sigma)| d \sigma\right] \\
& +\frac{\left(\left(\varrho_{2}-\varrho_{1}\right) v_{1}+\left(\varrho_{2}^{n-1}-\varrho_{1}^{n-1}\right) \varpi_{1}\right)}{\vartheta}\left[\lambda \int_{0}^{\varsigma}\left(\int_{0}^{\sigma} \frac{(\sigma-\theta)^{\delta-1}}{\Gamma(\delta)}|x(\theta)| d \theta\right) d \sigma\right. \\
& \left.+\mu \sum_{j=1}^{m-2} \xi_{j} \int_{0}^{\zeta_{j}} \frac{\left(\zeta_{j}-\sigma\right)^{\delta-1}}{\Gamma(\delta)}|x(\sigma)| d \sigma+\int_{0}^{1} \frac{(1-\sigma)^{\delta-1}}{\Gamma(\delta)}|x(\sigma)| d \sigma\right] \\
& \leq \frac{\|x\|}{\Gamma(\delta+1)}\left[\left[2\left(\varrho_{2}-\varrho_{1}\right)^{\delta}+\left(\varrho_{2}^{\delta}-\varrho_{1}^{\delta}\right)\right]+\frac{\rho\left(\left(\varrho_{2}-\varrho_{1}\right) v_{2}-\left(\varrho_{2}^{n-1}-\varrho_{1}^{n-1}\right) \varpi_{2}\right) \nu^{\delta-1}}{\vartheta \delta}\right. \\
& \left.+\frac{\left(\left(\varrho_{2}-\varrho_{1}\right) v_{1}+\left(\varrho_{2}^{n-1}-\varrho_{1}^{n-1}\right) \varpi_{1}\right)}{\vartheta}\left(\frac{\lambda \varsigma^{\delta+1}}{\delta+1}+\mu \sum_{j=1}^{m-2} \xi_{j} \zeta_{j}^{\delta}+1\right)\right]
\end{aligned}
$$

which is independent of $p$ and tends to zero as $\varrho_{2}-\varrho_{1} \rightarrow 0$. Thus, $\mathfrak{T}_{1}$ is equicontinuous. Step 4. The operator $\mathfrak{T}_{2}$ defined by (3.3) is continuous and $\Theta$ - contractive. To show the continuity of $\mathfrak{T}_{2}$ for $z \in \mathfrak{J}$, let us consider a sequence $p_{n}$ converging to $p$. Then we have

$$
\begin{aligned}
\left\|\mathfrak{T}_{2} p_{n}-\mathfrak{T}_{2} p\right\| & \leq\left[1+\frac{\left(z v_{1}+z^{n-1} \varpi_{1}\right)}{\vartheta}\left(\lambda \delta+\mu \sum_{j=1}^{m-2} \xi_{j}-1\right)\right]\left|\psi\left(p_{n}\right)-\psi(p)\right| \\
& \leq\left[1+\frac{\left(v_{1}+\varpi_{1}\right)}{\vartheta}\left(\lambda \delta+\mu \sum_{j=1}^{m-2} \xi_{j}+1\right)\right] \varepsilon\left\|p_{n}-p\right\|,
\end{aligned}
$$

which, in view of $\mathfrak{E}_{2}$, implies that $\mathfrak{T}_{2}$ is continuous. Also is $\mathfrak{T}_{2}$ is $\Theta$ - contractive, since

$$
\Theta=\left[1+\frac{\left(v_{1}+\varpi_{1}\right)}{\vartheta}\left(\lambda \delta+\mu \sum_{j=1}^{m-2} \xi_{j}+1\right)\right] \varepsilon=\widehat{\omega} \varepsilon<1 .
$$


Step 5. It remains to show that the set $\mathfrak{F}$ is bounded for every $\kappa$ !. Let $p \in \mathfrak{F}$ be a solution of the integral equation

$$
\begin{aligned}
p(z)= & \int_{0}^{z} \frac{\kappa(z-\sigma)^{\delta-1}}{\Gamma(\delta)} k(\sigma, p(\sigma)) d \sigma \\
& +\kappa\left[1+\frac{\left(z v_{1}+z^{n-1} \varpi_{1}\right)}{\vartheta}\left(\lambda \delta+\mu \sum_{j=1}^{m-2} \xi_{j}-1\right)\right] \psi(p) \\
& +\frac{\kappa \rho\left(z v_{2}-z^{n-1} \varpi_{2}\right)}{\vartheta}\left[\int_{0}^{\nu} \frac{(\nu-\sigma)^{\delta-2}}{\Gamma(\delta-1)} k(\sigma, p(\sigma)) d \sigma\right] \\
& +\frac{\kappa\left(z v_{1}+z^{n-1} \varpi_{1}\right)}{\vartheta}\left[\lambda \int_{0}^{\varsigma}\left(\int_{0}^{\sigma} \frac{(\sigma-\theta)^{\delta-1}}{\Gamma(\delta)} k(\theta, p(\theta)) d \theta\right) d \sigma\right. \\
& +\mu \sum_{j=1}^{m-2} \xi_{j} \int_{0}^{\zeta_{j}} \frac{\left(\zeta_{j}-\sigma\right)^{\delta-1}}{\Gamma(\delta)} k(\sigma, p(\sigma)) d \sigma \\
& \left.-\int_{0}^{1} \frac{(1-\sigma)^{\delta-1}}{\Gamma(\delta)} k(\sigma, p(\sigma)) d \sigma\right], \quad z \in \mathfrak{J}
\end{aligned}
$$

Then, for each $z \in \mathfrak{J}$, we have

$$
\begin{gathered}
|p(z)| \leq \int_{0}^{z} \frac{(z-\sigma)^{\delta-1}}{\Gamma(\delta)} x(\sigma) d \sigma+\kappa\left[1+\frac{\left(z v_{1}+z^{n-1} \varpi_{1}\right)}{\vartheta}\left(\lambda \delta+\mu \sum_{j=1}^{m-2} \xi_{j}-1\right)\right] \\
\times\left(\left|\psi\left(\frac{p(\sigma)}{\kappa}\right)-\psi(0)\right|+|\psi(0)|\right)+\frac{\rho\left(z v_{2}-z^{n-1} \varpi_{2}\right)}{\vartheta}\left[\int_{0}^{\nu} \frac{(\nu-\sigma)^{\delta-2}}{\Gamma(\delta-1)} x(\sigma) d \sigma\right] \\
+\frac{\left(z v_{1}+z^{n-1} \varpi_{1}\right)}{\vartheta}\left[\lambda \int_{0}^{\varsigma}\left(\int_{0}^{\sigma} \frac{(\sigma-\theta)^{\delta-1}}{\Gamma(\delta)} x(\theta) d \theta\right) d \sigma\right. \\
\left.+\mu \sum_{j=1}^{m-2} \xi_{j} \int_{0}^{\zeta_{j}} \frac{\left(\zeta_{j}-\sigma\right)^{\delta-1}}{\Gamma(\delta)} x(\sigma) d \sigma+\int_{0}^{1} \frac{(1-\sigma)^{\delta-1}}{\Gamma(\delta)} x(\sigma) d \sigma\right] \\
\quad \frac{(z-\sigma)^{\delta-1}}{\Gamma(\delta)} x(\sigma) d \sigma+\frac{\rho\left(z v_{2}-z^{n-1} \varpi_{2}\right)}{\vartheta}\left[\int_{0}^{\nu} \frac{(\nu-\sigma)^{\delta-2}}{\Gamma(\delta-1)} x(\sigma) d \sigma\right] \\
+\frac{\left(z v_{1}+z^{n-1} \varpi_{1}\right)}{\vartheta}\left[\lambda \int_{0}^{\varsigma}\left(\int_{0}^{\sigma} \frac{(\sigma-\theta)^{\delta-1}}{\Gamma(\delta)} x(\theta) d \theta\right) d \sigma\right. \\
\left.+\mu \sum_{j=1}^{m-2} \xi_{j} \int_{0}^{\zeta_{j}} \frac{\left(\zeta_{j}-\sigma\right)^{\delta-1}}{\Gamma(\delta)} x(\sigma) d \sigma+\int_{0}^{1} \frac{(1-\sigma)^{\delta-1}}{\Gamma(\delta)} x(\sigma) d \sigma\right] \\
+\left[1+\frac{\left(v_{1}+\varpi_{1}\right)}{\vartheta}\left(\lambda \delta+\mu \sum_{j=1}^{m-2} \xi_{j}+1\right)\right] \varepsilon\|p\|
\end{gathered}
$$

or

$$
(1-\widehat{\omega} \varepsilon)\|p\| \leq \int_{0}^{z} \frac{(z-\sigma)^{\delta-1}}{\Gamma(\delta)} x(\sigma) d \sigma+\frac{\rho\left(z v_{2}-z^{n-1} \varpi_{2}\right)}{\vartheta}\left[\int_{0}^{\nu} \frac{(\nu-\sigma)^{\delta-2}}{\Gamma(\delta-1)} x(\sigma) d \sigma\right]
$$




$$
\begin{gathered}
+\frac{\left(z v_{1}+z^{n-1} \varpi_{1}\right)}{\vartheta}\left[\lambda \int_{0}^{\varsigma}\left(\int_{0}^{\sigma} \frac{(\sigma-\theta)^{\delta-1}}{\Gamma(\delta)} x(\theta) d \theta\right) d \sigma\right. \\
\left.+\mu \sum_{j=1}^{m-2} \xi_{j} \int_{0}^{\zeta_{j}} \frac{\left(\zeta_{j}-\sigma\right)^{\delta-1}}{\Gamma(\delta)} x(\sigma) d \sigma+\int_{0}^{1} \frac{(1-\sigma)^{\delta-1}}{\Gamma(\delta)} x(\sigma) d \sigma\right] .
\end{gathered}
$$

Consequently, we have

$$
\begin{aligned}
\|p\| \leq & \mathfrak{V}:=\frac{1}{(1-\widehat{\omega} \varepsilon)}\left\{\int_{0}^{z} \frac{(z-\sigma)^{\delta-1}}{\Gamma(\delta)} x(\sigma) d \sigma\right. \\
& +\frac{\rho\left(z v_{2}-z^{n-1} \varpi_{2}\right)}{\vartheta}\left[\int_{0}^{\nu} \frac{(\nu-\sigma)^{\delta-2}}{\Gamma(\delta-1)} x(\sigma) d \sigma\right] \\
& +\frac{\left(z v_{1}+z^{n-1} \varpi_{1}\right)}{\vartheta}\left[\lambda \int_{0}^{\varsigma}\left(\int_{0}^{\sigma} \frac{(\sigma-\theta)^{\delta-1}}{\Gamma(\delta)} x(\theta) d \theta\right) d \sigma\right. \\
& +\mu \sum_{j=1}^{m-2} \xi_{j} \int_{0}^{\zeta_{j}} \frac{\left(\zeta_{j}-\sigma\right)^{\delta-1}}{\Gamma(\delta)} x(\sigma) d \sigma \\
& \left.\left.+\int_{0}^{1} \frac{(1-\sigma)^{\delta-1}}{\Gamma(\delta)} x(\sigma) d \sigma\right]\right\}
\end{aligned}
$$

which shows that the set $\mathfrak{F}$ is bounded, since $\widehat{\omega} \varepsilon<1$. Hence, $\mathfrak{T}$ has a fixed point in $\mathfrak{J}$ by Theorem 3.3, and consequently the problem (1.1)-(1.2) has a solution. This completes the proof.

Finally, we show that the existence of solutions for the boundary value problem (1.1)-(1.2) by applying a fixed poin theorem due to O'Regan.

Lemma 3.5. Denote by $\mathfrak{X}$ an open set in a closed, convex set $\mathfrak{A}$ of a Banach space $\mathfrak{H}$. Assume $0 \in \mathfrak{X}$. Also assume that $\mathfrak{T}(\widehat{\mathfrak{X}})$ is bounded and that $\mathfrak{T}: \widehat{\mathfrak{X}} \rightarrow \mathfrak{A}$ is given by $\mathfrak{T}=\mathfrak{T}_{1}+\mathfrak{T}_{2}$, in which $\mathfrak{T}_{1}: \widehat{\mathfrak{X}} \rightarrow \mathfrak{H}$ is a nonlinear contraction (i.e., there exists a nonnegative nondecreasing function $\varphi:[0, \infty) \rightarrow[0, \infty)$ satisfying $\vartheta(y)<y$ for $y>0$, such that $\left\|\mathfrak{T}_{2}(p)-\mathfrak{T}_{2}(q)\right\| \leq \vartheta(\|p-q\|) \forall p, q \in \widehat{\mathfrak{X}}$. Then, either

$\left(\mathfrak{W}_{1}\right) \mathfrak{T}$ has a fixed point $x \in \widehat{\mathfrak{X}} ;$ or

$\left(\mathfrak{W}_{2}\right)$ there exist a point $x \in \partial \mathfrak{X}$ and $\kappa \in(0,1)$ with $x=\kappa \mathfrak{T}(x)$, where $\widehat{\mathfrak{X}}$ and $\partial \mathfrak{X}$, respectively, represent the closure and boundary of $\mathfrak{X}$.

In the next result, we use the terminology:

$$
\Delta_{\theta}=\{p \in \mathfrak{G}:\|p\|<\theta\}, \mathfrak{V}_{\theta}=\max \{|k(z, p)|:(z, p) \in \mathfrak{J} \times[\theta,-\theta]\} .
$$

Theorem 3.6. The continuous function $k$ defined from $\mathfrak{J} \times \mathbb{R}$ to $\mathbb{R}$ and conditions $\left(\mathfrak{E}_{1}\right),\left(\mathfrak{E}_{2}\right),\left(\mathfrak{E}_{4}\right)$ hold. Also let us understand that:

$\left(\mathfrak{E}_{6}\right)$ there exists a nonnegative function $x \in C(\mathfrak{J}, \mathbb{R})$ and a nondecreasing function $\phi:[0, \infty) \rightarrow[0, \infty)$ such that $|k(z, v)| \leq x(z) \phi(\|v\|)$ for any $(z, v) \in \mathfrak{J} \times \mathbb{R} ;$

$\left(\mathfrak{E}_{7}\right) \sup _{\theta \in(0, \infty)} \frac{\theta}{\widehat{\eta} \phi(\theta)\|x\|}>\frac{1}{1-\widehat{\omega} \varepsilon}$, where $\widehat{\eta}$ and $\widehat{\omega}$ are defined in (3.4) and (3.5) respectively. Then the problem (1.1)-(1.2) has at least one solution on $\mathfrak{J}$. 
Proof. By the assumption $\left(\mathfrak{E}_{7}\right)$, there exists a number $\widehat{\theta}>0$ such that

$$
\frac{\widehat{\theta}}{\widehat{\eta} \phi(\widehat{\theta})\|x\|}>\frac{1}{1-\widehat{\omega} \varepsilon}
$$

We shall show that the operators $\mathfrak{T}_{1}$ and $\mathfrak{T}_{2}$ defined by (3.2) and (3.3) respectively, satisfy all the conditions of Lemma 3.5.

Step 1. The operator $\mathfrak{T}_{1}$ is continuous and completely continuous. We first show that $\mathfrak{T}_{1}\left(\Delta_{\widehat{\theta}}\right)$ is bounded. For any $p \in \Delta_{\widehat{\theta}}$, we have

$$
\begin{aligned}
& \left\|\mathfrak{T}_{1} p\right\| \leq \int_{0}^{z} \frac{(z-\sigma)^{\delta-1}}{\Gamma(\delta)}|k(\sigma, p(\sigma))| d \sigma \\
& +\frac{\rho\left(z v_{2}-z^{n-1} \varpi_{2}\right)}{\vartheta}\left[\int_{0}^{\nu} \frac{(\nu-\sigma)^{\delta-2}}{\Gamma(\delta-1)}|k(\sigma, p(\sigma))| d \sigma\right] \\
& +\frac{\left(z v_{1}+z^{n-1} \varpi_{1}\right)}{\vartheta}\left[\lambda \int_{0}^{\varsigma}\left(\int_{0}^{\sigma} \frac{(\sigma-\theta)^{\delta-1}}{\Gamma(\delta)}|k(\theta, p(\theta))| d \theta\right) d \sigma\right. \\
& +\mu \sum_{j=1}^{m-2} \xi_{j} \int_{0}^{\zeta_{j}} \frac{\left(\zeta_{j}-\sigma\right)^{\delta-1}}{\Gamma(\delta)}|k(\sigma, p(\sigma))| d \sigma \\
& \left.+\int_{0}^{1} \frac{(1-\sigma)^{\delta-1}}{\Gamma(\delta)}|k(\sigma, p(\sigma))| d \sigma\right] \\
& \leq \mathfrak{V}_{\theta} \int_{0}^{z} \frac{(z-\sigma)^{\delta-1}}{\Gamma(\delta)} x(\sigma) d \sigma \\
& +\frac{\mathfrak{V}_{\theta} \rho\left(z v_{2}-z^{n-1} \varpi_{2}\right)}{\vartheta}\left[\int_{0}^{\nu} \frac{(\nu-\sigma)^{\delta-2}}{\Gamma(\delta-1)} x(\sigma) d \sigma\right] \\
& +\frac{\mathfrak{V}_{\theta}\left(z v_{1}+z^{n-1} \varpi_{1}\right)}{\vartheta}\left[\lambda \int_{0}^{\varsigma}\left(\int_{0}^{\sigma} \frac{(\sigma-\theta)^{\delta-1}}{\Gamma(\delta)} x(\theta) d \theta\right) d \sigma\right. \\
& \left.+\mu \sum_{j=1}^{m-2} \xi_{j} \int_{0}^{\zeta_{j}} \frac{\left(\zeta_{j}-\sigma\right)^{\delta-1}}{\Gamma(\delta)} x(\sigma) d \sigma+\int_{0}^{1} \frac{(1-\sigma)^{\delta-1}}{\Gamma(\delta)} x(\sigma) d \sigma\right] \\
& \leq \frac{\|x\| \mathfrak{V}_{\theta}}{\Gamma(\delta+1)}\left[1+\frac{\rho\left|\left(v_{2}-\varpi_{2}\right)\right| \nu^{\delta-1}}{\vartheta \delta}\right. \\
& \left.+\frac{\left(v_{1}+\varpi_{1}\right)}{\vartheta}\left(\frac{\lambda \varsigma^{\delta+1}}{\delta+1}+\mu \sum_{j=1}^{m-2} \xi_{j} \zeta_{j}^{\delta}+1\right)\right] \\
& =\mathfrak{V}_{\theta}\|p\| \widehat{\eta} .
\end{aligned}
$$

Thus the operator $\mathfrak{T}_{1}\left(\mathfrak{V}_{\widehat{\theta}}\right)$ is uniformly bounded. Let $\varrho_{1}, \varrho_{2} \in \mathfrak{J}$ with $\varrho_{1}<\varrho_{2}$ and $p \in \mathfrak{B}_{\theta}$. Then

$$
\left|\left(\mathfrak{T}_{1} p\right)\left(\varrho_{2}\right)-\left(\mathfrak{T}_{1} p\right)\left(\varrho_{1}\right)\right| \leq \mathfrak{V}_{\theta}\left|\int_{0}^{\varrho_{1}} \frac{\left[\left(\varrho_{2}-\sigma\right)^{\delta-1}-\left(\varrho_{1}-\sigma\right)^{\delta-1}\right]}{\Gamma(\delta)} \times x(\sigma) d \sigma\right|
$$




$$
\begin{aligned}
& +\mathfrak{V}_{\theta}\left|\int_{\varrho_{1}}^{\varrho_{2}} \frac{\left(\varrho_{2}-\sigma\right)^{\delta-1}}{\Gamma(\delta)} x(\sigma) d \sigma\right| \\
& +\left|\frac{\mathfrak{V}_{\theta} \rho\left(\left(\varrho_{2}-\varrho_{1}\right) v_{2}-\left(\varrho_{2}^{n-1}-\varrho_{1}^{n-1}\right) \varpi_{2}\right)}{\vartheta}\right|\left[\int_{0}^{\nu} \frac{(\nu-\sigma)^{\delta-2}}{\Gamma(\delta-1)}|x(\sigma)| d \sigma\right] \\
& +\frac{\mathfrak{V}_{\theta}\left(\left(\varrho_{2}-\varrho_{1}\right) v_{1}+\left(\varrho_{2}^{n-1}-\varrho_{1}^{n-1}\right) \varpi_{1}\right)}{\vartheta}\left[\lambda \int_{0}^{\varsigma}\left(\int_{0}^{\sigma} \frac{(\sigma-\theta)^{\delta-1}}{\Gamma(\delta)}|x(\theta)| d \theta\right) d \sigma\right. \\
& \left.+\mu \sum_{j=1}^{m-2} \xi_{j} \int_{0}^{\zeta_{j}} \frac{\left(\zeta_{j}-\sigma\right)^{\delta-1}}{\Gamma(\delta)}|x(\sigma)| d \sigma+\int_{0}^{1} \frac{(1-\sigma)^{\delta-1}}{\Gamma(\delta)}|x(\sigma)| d \sigma\right] \\
& \leq \frac{\|x\| \mathfrak{V}_{\theta}}{\Gamma(\delta+1)}\left[\left[2\left(\varrho_{2}-\varrho_{1}\right)^{\delta}+\left(\varrho_{2}^{\delta}-\varrho_{1}^{\delta}\right)\right]+\frac{\rho\left(\left(\varrho_{2}-\varrho_{1}\right) v_{2}-\left(\varrho_{2}^{n-1}-\varrho_{1}^{n-1}\right) \varpi_{2}\right) \nu^{\delta-1}}{\vartheta \delta}\right. \\
& \left.+\frac{\left(\left(\varrho_{2}-\varrho_{1}\right) v_{1}+\left(\varrho_{2}^{n-1}-\varrho_{1}^{n-1}\right) \varpi_{1}\right)}{\vartheta}\left(\frac{\lambda \varsigma^{\delta+1}}{\delta+1}+\mu \sum_{j=1}^{m-2} \xi_{j} \zeta_{j}^{\delta}+1\right)\right]
\end{aligned}
$$

which is independent of $p$ and tends to zero as $\varrho_{2}-\varrho_{1} \rightarrow 0$. Thus, $\mathfrak{T}_{1}$ is equicontinuous. Hence, by the Arzela-Ascoli Theorem. $\mathfrak{T}_{1}\left(\mathfrak{V}_{\widehat{\theta}}\right)$ is a relatively compact set. Now, let $p_{n} \subset \mathfrak{V}_{\widehat{\theta}}$ with $\left\|p_{n}-p\right\| \rightarrow 0$. Then the $\left\|p_{n}(z)-p(z)\right\| \rightarrow 0$ is uniformly valid on $\mathfrak{J}$. From the uniform continuity of $k(z, p)$ on the compact set $\mathfrak{J} \times[\widehat{\theta},-\widehat{\theta}]$, it follows that

$$
\| k\left(z, p_{n}(z)\right)-k(z, p(z)) \mid \rightarrow 0
$$

uniformly on $\mathfrak{J}$. Hence $\left\|\mathfrak{T}_{1} p_{n}-\mathfrak{T}_{1} p\right\| \rightarrow 0$ as $n \rightarrow \infty$ which proves the continuity of $\mathfrak{T}_{1}$. This completes the proof Step 1.

Step 2. The operator $\mathfrak{T}_{2}: \mathfrak{V}_{\widehat{\theta}} \rightarrow C(\mathfrak{J}, \mathbb{R})$ is contractive. This is a consequence of $\left(\mathfrak{E}_{2}\right)$.

Step 3. The set $\mathfrak{T}\left(\mathfrak{V}_{\widehat{\theta}}\right)$ is bounded. The assumptions $\left(\mathfrak{E}_{2}\right)$ and $\left(\mathfrak{E}_{4}\right)$ imply that

$$
\left\|\mathfrak{T}_{2} p\right\| \leq \widehat{\omega} \varepsilon \widehat{\theta}
$$

for any $p \in \mathfrak{V}_{\widehat{\theta}}$. This, with the boundedness of the set $\mathfrak{T}_{1}\left(\mathfrak{V}_{\widehat{\theta}}\right)$ implies that the set $\mathfrak{T}\left(\mathfrak{V}_{\widehat{\theta}}\right)$ is bounded.

Step 4. Finally, it will be shown that the case $\mathfrak{W}_{2}$ in Lemma 3.5 does not hold. On the contrary, we suppose that $\mathfrak{W}_{2}$ holds. Then, we have that there exist $\kappa \in(0,1)$ and $p \in \partial \mathfrak{V}_{\widehat{\theta}}$ such that $p=\kappa \mathfrak{T} p$.

So, we have $\|p\|=\widehat{\theta}$ and

$$
\begin{gathered}
p(z)=\int_{0}^{z} \frac{\kappa(z-\sigma)^{\delta-1}}{\Gamma(\delta)} k(\sigma, p(\sigma)) d \sigma \\
+\kappa\left[1+\frac{\left(z v_{1}+z^{n-1} \varpi_{1}\right)}{\vartheta}\left(\lambda \delta+\mu \sum_{j=1}^{m-2} \xi_{j}-1\right)\right] \psi(p) \\
+\frac{\kappa \rho\left(z v_{2}-z^{n-1} \varpi_{2}\right)}{\vartheta}\left[\int_{0}^{\nu} \frac{(\nu-\sigma)^{\delta-2}}{\Gamma(\delta-1)} k(\sigma, p(\sigma)) d \sigma\right] \\
+\frac{\kappa\left(z v_{1}+z^{n-1} \varpi_{1}\right)}{\vartheta}\left[\lambda \int_{0}^{\varsigma}\left(\int_{0}^{\sigma} \frac{(\sigma-\theta)^{\delta-1}}{\Gamma(\delta)} k(\theta, p(\theta)) d \theta\right) d \sigma\right.
\end{gathered}
$$




$$
\left.+\mu \sum_{j=1}^{m-2} \xi_{j} \int_{0}^{\zeta_{j}} \frac{\left(\zeta_{j}-\sigma\right)^{\delta-1}}{\Gamma(\delta)} k(\sigma, p(\sigma)) d \sigma-\int_{0}^{1} \frac{(1-\sigma)^{\delta-1}}{\Gamma(\delta)} k(\sigma, p(\sigma)) d \sigma\right] \quad z \in \mathfrak{J}
$$

Using the assumptions $\left(\mathfrak{E}_{4}\right)-\left(\mathfrak{E}_{6}\right)$, we get

$$
\begin{aligned}
\widehat{\theta} \leq & \frac{\phi(\widehat{\theta})\|x\|}{\Gamma(\delta+1)}\left[1+\frac{\rho\left|\left(v_{2}-\varpi_{2}\right)\right| \nu^{\delta-1}}{\vartheta \delta}\right. \\
& \left.+\frac{\left(v_{1}+\varpi_{1}\right)}{\vartheta}\left(\frac{\lambda \varsigma^{\delta+1}}{\delta+1}+\mu \sum_{j=1}^{m-2} \xi_{j} \zeta_{j}^{\delta}+1\right)\right] \\
& +\widehat{\theta} \varepsilon\left[1+\frac{\left(v_{1}+\varpi_{1}\right)}{\vartheta}\left(\lambda \delta+\mu \sum_{j=1}^{m-2} \xi_{j}+1\right)\right] .
\end{aligned}
$$

which yields

$$
\widehat{\theta} \leq \widehat{\eta} \phi(\widehat{\theta})\|x\|+\widehat{\omega} \varepsilon
$$

Thus, we get a contradiction :

$$
\frac{\widehat{\theta}}{\widehat{\eta} \phi(\widehat{\theta})\|x\|} \leq \frac{1}{1-\widehat{\omega} \varepsilon} .
$$

Thus, the operators $\mathfrak{T}_{1}$ and $\mathfrak{T}_{2}$ satisfy all the conditions of Lemma 3.5. Hence, the operator $\mathfrak{T}$ has at least one fixed point $p \in \mathfrak{V}_{\widehat{\theta}}$, which is a solution of the problem (1.1)-(1.2). This completes the proof.

Example 3.7. Consider the fractional differential equation given by

$$
C_{\mathfrak{D}^{\frac{5}{2}}} p(z)=\frac{e^{-z}}{2 \sqrt{z^{2}+16}}\left(\frac{1}{2}+z \tan ^{-1}(z)\right), \quad z \in \mathfrak{J},
$$

supplemented with the boundary conditions of Example 3.2.

Observe that $|k(z, p)| \leq x(z) \phi(|p|)$ with

$$
x(z)=\frac{e^{-z}}{4 \sqrt{z^{2}+16}}, \phi(|p|)=1+|p|
$$

and $\psi(0)=0, \varepsilon=\frac{1}{10}$ as $|\psi(v)-\psi(w)| \leq \frac{1}{10}|v-w|$. With

$$
\phi(\theta)=1+\theta,\|x\|=\frac{1}{16}, \widehat{\eta} \cong 1.0683, \widehat{\omega} \cong 0.36416,
$$

we have that $\left(\mathfrak{E}_{7}\right)$ holds, since

$$
\frac{\widehat{\theta}}{\widehat{\eta} \phi(\widehat{\theta})\|x\|} \cong 14.9771>1.03779 \cong \frac{1}{1-\widehat{\omega} \varepsilon} .
$$

Thus, all the conditions of Theorem 3.6 is satisfied and here the problem (3.9) with (3.7) has at least one solution on $\mathfrak{J}$. 


\section{References}

[1] Agarwal, R.P., Ahmad, B., Garout, D., Alsaedi, A., Existence results for coupled nonlinear fractional differential equations equipped with nonlocal coupled flux and multi-point boundary conditions, Chaos Solitons Fractals, 2017, doi: 10.1016/j.chaos.2017.03.025.

[2] Ahmad, B., Alsaedi, A., Alsharif, A., Existence results for fractional-order differential equations with nonlocal multi-point-strip conditions involving Caputo derivative, Adv. Difference Equ., 2015, doi:10.1186/s13622-015-0684-3.

[3] Ahmad, B., Alsaedi, A., Garout, D., Existence results for Liouville-Caputo type fractional differential equations with nonlocal multi-point and sub-strips boundary conditions, Comput. Math. Appl., 2016, doi: 10.1016/j.camwa.2016.04.015.

[4] Ahmad, B., Ntouyas, S.K., Existence of solutions for fractional differential inclusions with nonlocal strip conditions, Arab J. Math. Sci., 18(2012), 121-134.

[5] Ahmad, B., Ntouyas, S.K., Existence results for nonlocal boundary value problems of fractional differential equations and inclusions with strip conditions, Bound. Value Probl., 2012, doi: 10.1186/1687-2770-2012-55.

[6] Ahmad, B., Ntouyas, S.K., Existence results for fractional differential inclusions with Erdelyi-Kober fractional integral conditions, An. Stiint. Univ. "Ovidius" Constanța Ser. Mat., 25(2017), no. 2, 5-24.

[7] Ahmad, B., Ntouyas, S.K., Agarwal, R.P., Alsaedi, A., Existence results for sequential fractional integrodifferential equations with nonlocal multi point and strip conditions, Fract. Calc. Appl. Anal., 18(2015), 261-280.

[8] Alipour, M., Baleanu, D., On the Kolmogorov forward equations with Caputo and Riemann-Liouville fractions derivatives, An. Stiint. Univ. "Ovidius" Constanţa Ser. Mat., 24, 2016, no. 3, 5-20.

[9] Deepmala, Agarwal, R.P., Existence and Uniqueness of solutions for certain functional equations and system of functional equations arising in dynamic programming, An. Stiint. Univ. "Ovidius" Constanţa Ser. Mat., 24(2016), no. 1, 3-28.

[10] Diethelm, K., The Analysis of Fractional Differential Equations, Springer, Berlin, Heidelberg, 2010.

[11] Ding, X., Ahmad, B., A generalized Volterra-Fredholm integral inequality and its applications to fractional differential equations, Adv. Difference Equ., 2018, doi: 10.1186/s13662-018-1548-4.

[12] Kilbas, A.A., Srivastava, H.M., Trujillo, J.J., Theory and Applications of Fractional Differential Equations, Amsterdam, Boston, Elsevier, 2006.

[13] Klafter, J., Lim, S.C., Metzler, R., Fractional Dynamics: Recent Advances, World Scientific, 2012.

[14] Miller, K.S., Ross, B., An Introduction to the Fractional Calculus and Fractional Differential Equations, Wiley, New York, 1993.

[15] Nyamoradi, N., Zhou, Y., Tayyebi, E., Ahmad, B., Alsaedi, A., Nontrivial solutions for time fractional nonlinear Schrodinger-Kirchhoff type equations, Discrete Dyn. Nat. Soc., 2017, Art. ID 9281049, 9 pages, doi: 10.1155/2017/9281049.

[16] Podlubny, I., Fractional Differential Equations: An Introduction to Fractional Derivatives, Fractional Differential Equations, Some Methods of Their Solution and Some of Their Applications, Academic Press, San Diego - Boston - New York - London - Tokyo - Toronto, 1999. 
[17] Sabatier, J., Agrawal, O.P., Tenreiro Machado, J.A., Advances in Fractional Calculus: Theoretical Developments and Applications in Physics and Engineering, Springer Netherlands, 2007.

[18] Zhou, Y., Ahmad, B., Wei He, J., Alsaedi, A., Existence and attractivity of fractional evolution equations, Discrete Dyn. Nat. Soc., 2018, Art. ID 1070713, 9 pages.

Muthaiah Subramanian

Department of Mathematics

Sri Ramakrishna Mission Vidyalaya College of Arts and Science

Coimbatore - 641 020, Tamilnadu, India

e-mail: subramanianmcbe@gmail.com

A Ramamurthy Vidhya Kumar

Department of Mathematics

Sri Ramakrishna Mission Vidyalaya College of Arts and Science

Coimbatore - 641 020, Tamilnadu, India

e-mail: vidhu.ar@gmail.com

Thangaraj Nandha Gopal

Department of Mathematics

Sri Ramakrishna Mission Vidyalaya College of Arts and Science

Coimbatore - 641 020, Tamilnadu, India

e-mail: nandhu792002@yahoo.co.in 